\title{
Hemophagocytic Lymphohistiocytosis
}

National Cancer Institute

\section{Source}

National Cancer Institute. Hemophagocytic Lymphohistiocytosis. NCI Thesaurus. Code C34792.

A rare but potentially life-threatening disorder characterized by the proliferation of histiocytes and macrophages and phagocytosis of red blood cells, white blood cells, and platelets. It may be inherited or secondary, due to infections, autoimmune disorders, or underlying malignancies. Signs and symptoms include fever, lymphadenopathy, hepatomegaly, splenomegaly, and pancytopenia. 